\title{
$p$-Wave Polaron
}

\author{
Jesper Levinsen, ${ }^{1, *}$ Pietro Massignan, ${ }^{2}$ Frédéric Chevy, ${ }^{3}$ and Carlos Lobo ${ }^{4}$ \\ ${ }^{1}$ T.C.M. Group, Cavendish Laboratory, JJ Thomson Avenue, Cambridge CB3 OHE, United Kingdom \\ ${ }^{2}$ ICFO_The Institute of Photonic Sciences-08860 Castelldefels (Barcelona), Spain \\ ${ }^{3}$ Laboratoire Kastler Brossel-ENS, Université Paris 6, CNRS, 24 rue Lhomond, 75005 Paris, France \\ ${ }^{4}$ School of Mathematics, University of Southampton, Highfield, Southampton, SO17 1BJ, United Kingdom
}

(Received 10 April 2012; published 16 August 2012)

\begin{abstract}
We consider a single impurity immersed in a Fermi sea close to an interspecies $p$-wave Feshbach resonance. We calculate its dispersion and spectral response to a radio frequency pulse. In a magnetic field, dipolar interactions split the resonance and two novel features with respect to the $s$-wave case appear: a third polaron branch in the excitation spectrum, in addition to the usual attractive and repulsive ones; and an anisotropic dispersion of the impurity characterized by different effective masses perpendicular and parallel to the magnetic field. The anisotropy can be tuned as a function of the field strength and the two effective masses may have opposite signs, or become smaller than the bare mass.
\end{abstract}

DOI: 10.1103/PhysRevLett.109.075302

PACS numbers: 67.85.Lm, 05.30.Fk

Understanding the physics of a single impurity in a degenerate ultracold gas has been essential in discovering the phase diagram of spin-imbalanced Fermi mixtures [1-3]. A large effort has been devoted to its study, resulting in an impressive agreement between theory and experiment [4]. The emergent picture is that the quasiparticle formed by the impurity interacting with the background gas can be fermionic (a "polaron") or bosonic (a "molecule").

So far, the polaron has not been studied close to higher partial wave resonances. Particularly interesting are $p$-wave resonances, as $p$-wave coupled superfluids are predicted to display a richer phase diagram than their $s$-wave counterparts [5-8]. For example, the BardeenCooper-Schrieffer (BCS) and Bose-Einstein condensation (BEC) regimes of these superfluids are separate phases [9], and each of these can be either chiral or polar [7]. For ${ }^{6} \mathrm{Li}$ and ${ }^{40} \mathrm{~K}, p$-wave resonances between atoms in the same hyperfine state were found to have a narrow magnetic width, of order $\lesssim 1 \mathrm{G}[10,11]$. However, $p$-wave resonances in $\mathrm{Li}-\mathrm{K}$ mixtures [12] proved to have larger widths, in the range 1-10 G. Given the stability of magnetic fields in state of the art experiments $(\approx \pm 1 \mathrm{mG})$, such resonances are now finally accessible for detailed study.

Here we study the $p$-wave polaron problem by considering a single impurity atom (" $\downarrow$ "), immersed in a $\uparrow$ Fermi sea, both species having the same mass and strong interspecies $p$-wave interactions.

An interesting feature of $p$-wave Feshbach resonances is that they are generally split into a doublet according to the projection $m_{l}$ of the relative angular momentum onto the magnetic field axis. As first discussed in Ref. [11], the $m_{l}= \pm 1$ resonances are shifted towards higher energy from the $m_{l}=0$ resonance by the magnetic dipole-dipole interaction in the presence of the external magnetic field. As we shall see, an important consequence is that when the resonance splitting is large enough, a new polaron branch appears between the attractive and repulsive polaron branches which had been previously observed close to $s$-wave Feshbach resonances [13-15]. This is reflected in the spectral function of the impurity atom displayed in Fig. 1. Additionally, the polaron's effective mass is generally anisotropic, and can have different signs parallel to and perpendicular to the magnetic field. Clearly, these two new features could lead to many-body behavior (e.g., collective
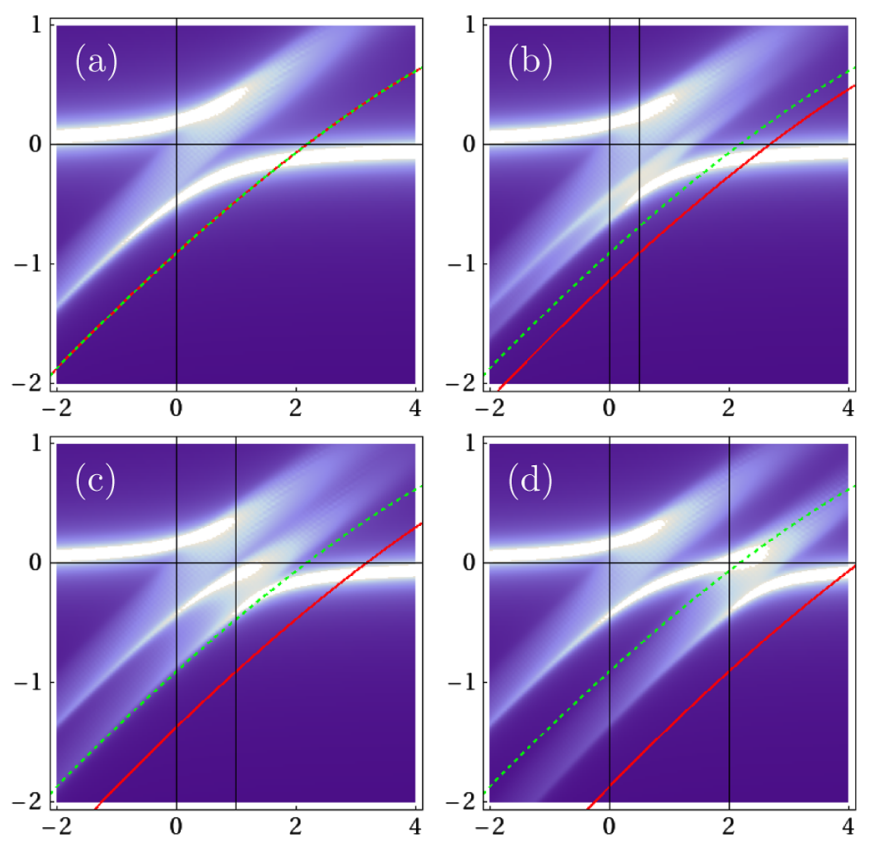

FIG. 1 (color online). Spectral function of the $p$-wave polaron. The horizontal and vertical axes are $-\left(k_{F}^{3} v_{ \pm 1}\right)^{-1}$ and $\omega / \epsilon_{F}$. The $m_{l}= \pm 1$ and $m_{l}=0$ resonances are located respectively at $x=0$ and $x=\delta$, with $\delta=0$ (a), 0.5 (b), 1 (c), 2 (d). Thick lines are the dressed molecules with $m_{l}=0$ (solid line) and $m_{l}= \pm 1$ (dashed line). Here $-k_{0} / k_{F}=10$. 
modes, possible superfluid states) which would be dramatically different from that of the $s$-wave system.

The impurity may also bind to a particle from the Fermi sea to form a diatomic molecule, and as the interactions are increased this molecular state becomes energetically favorable. We calculate the energies of the polaron and the molecule, and show that the position of the polaronmolecule transition shifts towards the BCS side for increasingly narrow resonances.

To model the $p$-wave Feshbach resonance we use a twochannel Hamiltonian previously introduced in the context of identical fermions interacting close to a $p$-wave resonance [7,16] (we set $\hbar=1)$ :

$$
\begin{aligned}
H= & \sum_{\mathbf{p}, \sigma=\uparrow, \downarrow} \frac{p^{2}}{2 m} a_{\sigma \mathbf{p}}^{\dagger} a_{\sigma \mathbf{p}}+\sum_{\mathbf{q}, \mu}\left(\epsilon_{\mu}+\frac{q^{2}}{4 m}\right) b_{\mu \mathbf{q}}^{\dagger} b_{\mu \mathbf{q}} \\
& +\sum_{\mathbf{p}, \mathbf{q}, \mu} \frac{g(|\mathbf{p}|)}{\sqrt{V}} \mathbf{p}_{\mu}\left(b_{\mathbf{q} \mu}^{\dagger} a_{\uparrow(\mathbf{q} / 2)+\mathbf{p}} a_{\downarrow(\mathbf{q} / 2)-\mathbf{p}}\right. \\
& \left.+a_{\downarrow(\mathbf{q} / 2)-\mathbf{p}}^{\dagger} a_{\uparrow(\mathbf{q} / 2)+\mathbf{p}}^{\dagger} b_{\mathbf{q} \mu}\right) .
\end{aligned}
$$

We define bosonic operators $b_{\mu=x, y, z}$ in terms of the closed channel $l=1$ molecule operators $b_{m_{l}=0, \pm 1}$ as $b_{x} \equiv$ $\left(b_{+1}+b_{-1}\right) / \sqrt{2}, b_{y} \equiv-i\left(b_{+1}-b_{-1}\right) / \sqrt{2}, b_{z} \equiv b_{0}$. We specialize to the case where the $m_{l}= \pm 1$ resonances are degenerate [17]. $a_{\sigma}$ and $a_{\sigma}^{\dagger}$ are creation and annihilation operators of fermions with mass $m$, with $V$ the system volume. The resonance splitting caused by dipolar anisotropy may be modeled by a positive shift of the $m_{l}= \pm 1$ molecule energies: $\epsilon_{x, y}=\epsilon_{z}+\delta_{0}$ with $\epsilon_{x, y}=\epsilon_{ \pm 1}$ and $\epsilon_{z}=\epsilon_{0}$. In current experiments, $\delta_{0} \sim 0.5-5 \mu \mathrm{K}[11,16]$ which should be compared with the Fermi energy, typically of order $1 \mu \mathrm{K}$.

Interactions couple the closed channel molecule to a pair of atoms in the open channel, and are described by the coupling constant $g(|\mathbf{p}|) \mathbf{p}_{\mu}$ which vanishes above a cutoff $\Lambda$ of the order of the inverse van der Waals length. In the low energy limit, the results should not depend on the actual shape of $g$ and we choose the cutoff function to be a step function, $g(|\mathbf{p}|)=g \Theta(\Lambda-p)$.

Without a Fermi sea, the bare propagator of the closed channel molecule reads

$$
D_{\mu \nu}^{\mathrm{cl}}(\mathbf{p}, \omega)=\frac{\delta_{\mu \nu}}{\omega-\epsilon_{\mu}-p^{2} / 4 m+i 0} \equiv D_{\mu}^{\mathrm{cl}}(\mathbf{p}, \omega) \delta_{\mu \nu} .
$$

The renormalization of $p$-wave interactions proceeds similarly to the case of identical fermions [7]: The renormalized molecular propagator $D_{\mu \nu}^{0}$ is dressed by the polarization $\Pi^{0}$ as shown in Fig. 2(a). In vacuum $\Pi_{\mu \nu}^{0} \equiv$ $\Pi^{0} \delta_{\mu \nu}$ is diagonal and takes the form $\Pi^{0}(\mathbf{p}, \omega)=$ $\frac{1}{3} \int \frac{d^{3} q}{(2 \pi)^{3}} \frac{q^{2} g^{2}(q)}{\omega-q^{2} / m-p^{2} / 4 m+i 0}$. Thus the propagator in vacuum is also diagonal, i.e., $D_{\mu \nu}^{0}(\mathbf{p}, \omega)=\delta_{\mu \nu} /\left\{\left[D_{\mu}^{\mathrm{cl}}(\mathbf{p}, \omega)\right]^{-1}-\right.$ $\left.\Pi^{0}(\mathbf{p}, \omega)\right\}$. (a)

(b)
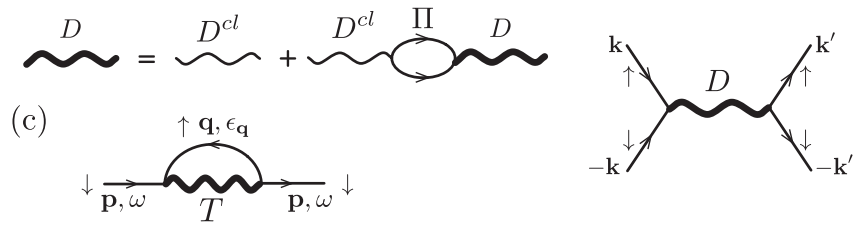

FIG. 2. (a) Renormalized propagator of molecules (thick line). Thin wavy lines are bare molecules, straight lines are fermions. (b) The diagram which leads to the $T$ matrix. (c) Self-energy of the impurity.

The elastic scattering between a spin- $\uparrow$ and a spin- $\downarrow$ atom in the ladder approximation is described by a $T$ matrix given by $T\left(\mathbf{k}, \mathbf{k}^{\prime}\right)=\sum_{\mu} g^{2}(k) k_{\mu} k_{\mu}^{\prime} D_{\mu \mu}^{0}\left(\mathbf{0}, k^{2} / m\right)$ [see Fig. 2(b)]. For identical fermions, the $T$ matrix was obtained in Ref. [7]. Note that the splitting of the $p$-wave resonance by the dipole-dipole interaction in the presence of a magnetic field breaks rotational symmetry. In order to relate the parameters of the model to physical observables, we compare with the low energy expansion of the $p$-wave scattering amplitude $f\left(\mathbf{k}, \mathbf{k}^{\prime}\right) \equiv-(m / 4 \pi) T\left(\mathbf{k}, \mathbf{k}^{\prime}\right)$ :

$$
f\left(\mathbf{k}, \mathbf{k}^{\prime}\right) \approx \sum_{\mu} \frac{k_{\mu} k_{\mu}^{\prime}}{-v_{\mu}^{-1}+\frac{1}{2} k_{0} k^{2}-i k^{3}} .
$$

Here, $v_{\mu}$ is the state dependent scattering volume and $k_{0}$, with dimension of momentum, is the $p$-wave analogue of the effective range [18]. Evaluating $\Pi^{0}$ yields $v_{\mu}=$ $-\frac{m g^{2}}{12 \pi}\left(\epsilon_{\mu}-\frac{m g^{2} \Lambda^{3}}{18 \pi^{2}}\right)^{-1}$ and $k_{0}=-\frac{24 \pi}{m^{2} g^{2}}\left(1+c_{2}\right)$ with $c_{2} \equiv$ $m^{2} g^{2} \Lambda / 6 \pi^{2}$. The vacuum molecule propagator is then

$$
D_{\mu \nu}^{0}(\mathbf{p}, \omega)=\frac{12 \pi /\left(m g^{2}\right) \delta_{\mu \nu}}{v_{\mu}^{-1}-\frac{1}{2} k_{0}\left(m \omega-\frac{p^{2}}{4}\right)+\left(\frac{p^{2}}{4}-m \omega-i 0\right)^{3 / 2}} .
$$

$k_{0}$ is naturally large and negative, of order $\Lambda[19,20]$.

$p$-wave resonances in the many-body system are characterized by two dimensionless parameters. Of these $\gamma \sim\left(1+c_{2}\right) k_{F} / k_{0}$ controls interactions at the scale of the Fermi momentum $k_{F}$ (i.e., many-body physics). Resonances with $\gamma \ll 1 \quad(\gamma \gg 1)$ are termed narrow (wide) and quantum fluctuations are suppressed for $\gamma \ll 1$ [7]. The parameter $c_{2}$ controls few-body physics [19] and distinguishes strongly coupled $\left(c_{2} \gg 1\right)$ from weakly coupled $\left(c_{2} \ll 1\right)$ systems. Identical fermions interacting close to a strong Feshbach resonance may form trimer states [20]. However, using the method of Ref. [19], we have checked that trimers consisting of two identical fermions and an impurity of equal mass do not form in vacuum in the absence of resonance splitting.

We now turn to the question of the many-body state of a spin- $\downarrow$ impurity immersed in a spin- $\uparrow$ Fermi sea at zero temperature. In the $s$-wave case both the attractive and repulsive polaron branches are accurately described by dressing the impurity with a single particle-hole 
excitation [2,21]; we will adopt the same approximation for the $p$-wave case. Indeed, this approximation becomes exact far away from resonance, or in the limit of narrow resonances [7], and provides a rigorous upper bound to the energy, as it corresponds to a variational ansatz. The propagator of the impurity with momentum $\mathbf{p}$ and energy $\omega$ in the medium is $G_{\downarrow}(\mathbf{p}, \omega)=\left[\omega-p^{2} / 2 m-\right.$ $\Sigma(\mathbf{p}, \omega)+i 0]^{-1}$ with $\Sigma$ the self-energy. The energy of the polaron satisfies $E=\operatorname{Re}[\Sigma(\mathbf{p}, E)]$, provided the associated imaginary part is small compared to $E$. The selfenergy in the single particle-hole approximation is given by the diagram in Fig. 2(c):

$$
\Sigma(\mathbf{p}, \omega)=\int \frac{d^{3} \mathbf{q}}{(2 \pi)^{3}} n_{F \uparrow}(\mathbf{q}) T\left(\mathbf{p}, \omega ; \mathbf{q}, \boldsymbol{\epsilon}_{\mathbf{q}}\right),
$$

where the Fermi function $n_{F \uparrow}(\mathbf{q})$ takes the value 1 if the state with momentum $\mathbf{q}$ is occupied, 0 otherwise. The offshell $T$ matrix in the medium is

$$
\begin{aligned}
T\left(\mathbf{p}, \omega ; \mathbf{q}, \boldsymbol{\epsilon}_{\mathbf{q}}\right)= & g^{2}(|\mathbf{p}-\mathbf{q}| / 2) \sum_{\mu \nu}\left(\frac{\mathbf{p}-\mathbf{q}}{2}\right)_{\mu}\left(\frac{\mathbf{p}-\mathbf{q}}{2}\right)_{\nu} \\
& \times\left\{\left[D^{\mathrm{cl}}\left(\mathbf{p}+\mathbf{q}, \omega+\boldsymbol{\epsilon}_{\mathbf{q}}\right)\right]^{-1}\right. \\
& \left.-\Pi\left(\mathbf{p}+\mathbf{q}, \omega+\epsilon_{\mathbf{q}}\right)\right\}_{\mu \nu}^{-1} .
\end{aligned}
$$

Here $(\mathbf{p}, \omega)\left[\left(\mathbf{q}, \epsilon_{\mathbf{q}}\right)\right]$ are the $\uparrow[\downarrow]$ atom momentum and energy entering the $T$ matrix, with $\epsilon_{\mathbf{q}}=q^{2} / 2 m$. The polarization in the medium is given by

$\Pi_{\mu \nu}(\mathbf{q}, \omega)=\int \frac{d^{3} \mathbf{k}}{(2 \pi)^{3}} \frac{k_{\mu} k_{\nu} g^{2}(k)\left[1-n_{F \dagger}(\mathbf{k}+\mathbf{q} / 2)\right]}{\omega-q^{2} / 4 m-k^{2} / m+i 0}$.

The polarization is a tensor, as collisions with particles in the Fermi sea generally do not preserve the projection of the molecule's angular momentum. However, the matrix inverse in Eq. (5) may be performed as follows: Decompose $\quad \Pi_{\mu \nu}\left(\mathbf{p}+\mathbf{q}, \omega+\epsilon_{\mathbf{q}}\right) \equiv A \delta_{\mu \nu}+B(\mathbf{p}+\mathbf{q})_{\mu} \times$ $(\mathbf{p}+\mathbf{q})_{\nu} /|\mathbf{p}+\mathbf{q}|^{2}$. Then sums containing only $A$ or only $B$ terms form geometric series. Resumming first the $A$ terms, and successively adding the $B$ terms, one finds

$$
T\left(\mathbf{p}, \omega ; \mathbf{q}, \boldsymbol{\epsilon}_{\mathbf{q}}\right)=\frac{g^{2}}{4}\left[\mathcal{D}_{-}+\frac{\mathcal{D}_{\mathrm{x}}^{2}}{|\mathbf{p}+\mathbf{q}|^{2} / B-\mathcal{D}_{+}}\right],
$$

with $\quad \mathcal{D}_{ \pm} \equiv \sum_{\mu} \frac{(\mathbf{p} \pm \mathbf{q})_{\mu}^{2}}{\left[D_{\mu}^{\mathrm{cl}}\left(\mathbf{p}+\mathbf{q}, \omega+\epsilon_{\mathbf{q}}\right)\right]^{-1}-A} \quad$ and $\quad \mathcal{D}_{\mathrm{x}} \equiv$ $\sum_{\mu} \frac{(\mathbf{p}+\mathbf{q})_{\mu}(\mathbf{p}-\mathbf{q})_{\mu}}{\left[D_{\mu}^{\mathrm{c}}\left(\mathbf{p}+\mathbf{q}, \omega+\epsilon_{\mathbf{q}}\right)\right]^{-1}-A}$.

The above formalism allows us to calculate the spectral function of the impurity atoms, defined as $A_{\downarrow}(\mathbf{p}, \omega)=$ $-2 \operatorname{Im}\left[G_{\downarrow}(\mathbf{p}, \omega)\right]$. Within linear response theory, the spectral function coincides with the response of the system to a low-power radio frequency pulse, which transfers impurities from a noninteracting to the strongly interacting state $[13,14,22,23]$. By this method, one effectively adds only a few impurity atoms to the Fermi sea, probing precisely the single particle spectral function $A_{\downarrow}$.
In particular, $A_{\downarrow}$ peaks at the energy of the quasiparticle states with a finite wave function overlap with the bare impurity, i.e., the polarons. Our results for the spectral function close to resonance are shown in Fig. 1 for several values of the dimensionless resonance splitting $\delta \equiv$ $\delta_{0} 12 \pi /\left(m g^{2} k_{F}^{3}\right)$. For vanishing $\delta_{0}$, the picture is qualitatively the same as in the $s$-wave case [2,22-24] with an attractive (repulsive) polaron of energy lower (higher) than the bare impurity. However, an additional branch appears for finite resonance splitting. For small resonance splitting, $\delta_{0} \lesssim \epsilon_{F}$, the intermediate branch disappears in the $m_{l}=0$ molecule-hole continuum. For larger $\delta_{0}$ the intermediate branch shows up as an isolated spectral line which is a repulsive polaron close to the $m_{l}=0$ resonance, approximately a free impurity between the resonances, and an attractive polaron close to the $m_{l}= \pm 1$ resonance. It should be noted that our ansatz does not contain all possible decay processes for the polaron or molecule. For example, a process which occurs at zero momentum involves the decay of a polaron into a molecule, two holes and one particle $[22,23]$. This has the effect of giving the lower polaron branch a finite lifetime when it is no longer the ground state and of generally increasing the width of the spectral lines of the excited quasiparticles in Fig. 1.

In addition to energy, the polaron is characterized by its quasiparticle weight, the residue, given by $Z=\left\{1-\partial_{\omega} \operatorname{Re}[\Sigma(\mathbf{0}, \omega)]\right\}^{-1}$ evaluated at the quasiparticle energy. In Fig. 3 we display the energy and residue across the resonance for various values of $k_{0} / k_{F}$. We see that $|E|$ decreases for increasing $\left|k_{0} / k_{F}\right|$ as expected since particlehole fluctuations become suppressed [7]. In this limit, Eq. (4) in the absence of resonance splitting becomes

$$
\Sigma(\mathbf{0}, E)=-\int_{0}^{k_{F}} \frac{3 q^{4} /(2 \pi m) d q}{-\boldsymbol{v}^{-1}+\frac{1}{2} m k_{0}\left(E+\epsilon_{\mathbf{q}} / 2\right)-i 0} .
$$

As mentioned above, the impurity may also bind a particle from the majority Fermi sea to form a bosonic quasiparticle-a molecule. In the "Cooper pair" approximation (i.e., with no particle-hole pairs), the energy $E_{\text {mol }}$ of

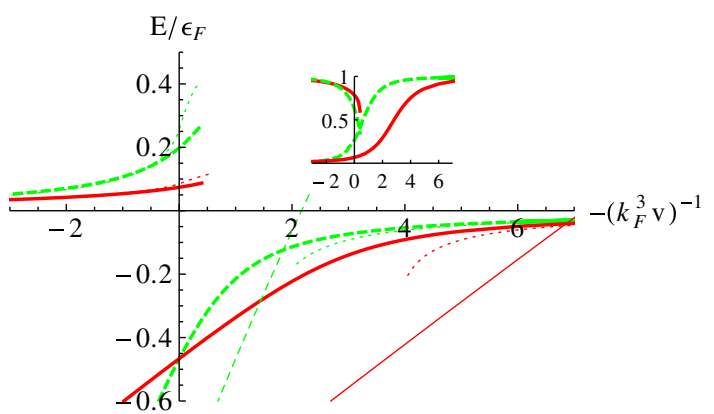

FIG. 3 (color online). Energy (main) and residue (inset) of the two $p$-wave polarons for $\delta=0$ through the crossover for range parameters: $-k_{0} / k_{F}=30$ (solid line), 10 (dashed line). Dotted lines are the perturbative result, Eq. (8), and thin lines are the molecule energies as given by the Thouless pole. 


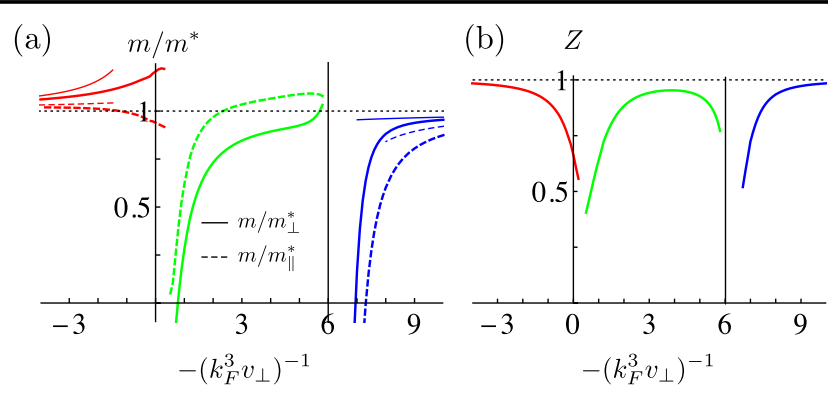

FIG. 4 (color online). (a) Inverse effective mass of $p$-wave polarons moving perpendicular (solid lines) and parallel (dashed lines) to the magnetic field. Here $-k_{0} / k_{F}=10$, and the two resonances are split by $\delta=6$, i.e., the $m_{l}= \pm 1 \quad\left(m_{l}=0\right)$ resonance lies at $x=0(x=6)$. Thin lines are the analytic weak-coupling result, valid when $\left|v_{\perp, \|} k_{F}^{3}\right|^{-1} \gg\left|k_{0} / k_{F}\right|$. (b) Polaron residues.

the molecular state is given by the pole of the $T$ matrix at $\mathbf{p}=0$ and $\omega=E_{\mathrm{mol}}+\epsilon_{F}$. The latter approximation is an upper bound to the real energy of the molecule, which becomes exact for $\left|k_{0} / k_{F}\right| \gg 1$. On the BEC side, $E_{\text {mol }}$ tends to $E_{b}-\epsilon_{F}$, with $E_{b}$ the energy of the molecule in vacuum. The molecule energy is included in Figs. 1 and 3. The latter illustrates that for increasing $-k_{0} / k_{F}$ the transition from a polaronic to a molecular ground state takes place further towards the BCS limit, as is the case close to a narrow $s$-wave Feshbach resonance [13,25-27]. Note, however, that the limit of narrow $p$-wave resonances corresponds to small densities, while the opposite is true for $s$-wave resonances.

The presence of a magnetic field which splits the resonances also breaks isotropy since it differentiates between $m_{l}$ levels. One consequence is the appearance of a strongly anisotropic dispersion of the impurity close to either of the resonances. At small momenta, the dispersion may be written as $E(\mathbf{p})=E(\mathbf{0})+p_{\|}^{2} / 2 m_{\|}^{*}+p_{\perp}^{2} / 2 m_{\perp}^{*}$, where $p_{\|}$ and $p_{\perp}$ are the projections of $\mathbf{p}$ along the magnetic field, and on the plane perpendicular to it. We compute the effective mass tensor, showing how, on the BEC side, its magnitude is generally smaller than the bare mass of the particle, as follows from the analytic weak-coupling result $m / m_{\perp, \|}^{*} \approx 1+v_{\perp, \|} k_{F}^{3} / \pi$. This is allowed since, in this region, the polaron is no longer the ground state. Moreover, close to the $m_{l}=0$ resonance $m / m_{\|}^{*}$ becomes negative while $m / m_{\perp}^{*}$ is still positive, the opposite behavior occurring in the vicinity of the $m_{l}= \pm 1$ resonance. This is illustrated in Fig. 4.

We now make a few final remarks. In the above, any background $s$-wave scattering between the two atomic species has been ignored. This is justified provided the background scattering is small, as is the case for a $p$-wave resonance close to a zero crossing of an $s$-wave resonance. At vanishing energy the $s$-wave channel dominates. However, in the present system the low energy scale is set by $\epsilon_{F}$. The total cross section for two atoms in the $s$-wave channel is $\sigma_{s}=4 \pi a^{2}$ while the $p$-wave cross section is $\sigma_{p}=12 \pi\left|f_{p}\left(k_{F}\right)\right|^{2}$ [28]. If $\left|v k_{F}^{3}\right|^{-1} \gg\left|k_{0} / k_{F}\right|$ then $p$-wave scattering is dominant for $\left|v k_{F}^{3}\right| \gtrsim\left|k_{F} a\right|$ while in the opposite case one finds $\left|k_{0} a\right| \lesssim 1$. Both requirements are achievable provided $k_{0}$ is not too large.

Early experimental studies of $p$-wave Feshbach molecules showed that these were short-lived, with lifetimes of 2 to $20 \mathrm{~ms}$ [10,29-31]. This was explained in Refs. [19,20,32] as due to relaxation processes and recombination into trimers. However, radio frequency spectroscopy has been able to study the complete spectral response of metastable many-body states of strongly interacting fermions with an intrinsic lifetime of only $30 \mathrm{~ms}$ [13] including their polaronic and molecular branches. Furthermore, a recent proposal to modify the $p$-wave interaction by coupling to a long-range excited state with the use of an optical Feshbach resonance predicts the possibility of suppressing three-body recombination [33], potentially allowing for longer lifetimes.

The polaron problem provides access to the equation of state of an imbalanced Fermi gas in its normal phase, as the latter may generally be written as the energy of an ideal gas of quasiparticles [1]. The generalization to split $p$-wave resonances yields

$$
\frac{E}{N_{\uparrow}}=\frac{3}{5} \epsilon_{F}\left[1+\frac{m\left(N_{\downarrow} / N_{\uparrow}\right)^{5 / 3}}{\left(m_{\perp}^{*}\right)^{2 / 3}\left(m_{\|}^{*}\right)^{1 / 3}}\right]+\frac{N_{\downarrow}}{N_{\uparrow}} E_{p}+\ldots,
$$

where $E_{p}$ and $m^{*}$ are the polaron energy and effective mass on the branch of interest and $N_{\uparrow, \downarrow}$ the number of spin- $\uparrow, \downarrow$ particles.

In this Letter, we studied the properties of the $p$-wave polaron. These are observable by radio frequency spectroscopy and angle-resolved photoemission spectroscopy. The formalism presented here may be extended to heteronuclear Fermi-Fermi mixtures. In the presence of a mass imbalance, we expect the polaron-molecule crossing to shift towards the BCS side for heavy impurities, while trimers may arise for sufficiently light impurities. Our results are also relevant to studies of resonantly enhanced atom-dimer scattering near the appearance of confinement induced $p$-wave trimers [34,35], in which case the dimer acts as the impurity investigated here.

We are grateful to V. Gurarie, M. Lewenstein, and D. S. Petrov for fruitful discussions. J. L. acknowledges support from a Marie Curie Intra European grant within the 7th European Community Framework Programme. P. M. acknowledges funding through MEC project TOQATA and ERC Advanced Grant QUAGATUA. C. L. acknowledges support from the EPSRC through Contract No. EP/ E053033/1. J.L., P. M., and C.L. wish to thank the Aspen Center for Physics, where part of this work was realized. F. C. acknowledges support from Région Ile de France (IFRAF), Institut Universitaire de France and ERC (Advanced Grant Ferlodim). 
*jfl36@cam.ac.uk

[1] C. Lobo, A. Recati, S. Giorgini, and S. Stringari, Phys. Rev. Lett. 97, 200403 (2006).

[2] F. Chevy, Phys. Rev. A 74, 063628 (2006).

[3] S. Pilati and S. Giorgini, Phys. Rev. Lett. 100, 030401 (2008).

[4] F. Chevy and C. Mora, Rep. Prog. Phys. 73, 112401 (2010).

[5] V. Gurarie, L. Radzihovsky, and A. V. Andreev, Phys. Rev. Lett. 94, 230403 (2005).

[6] C.-H. Cheng and S.-K. Yip, Phys. Rev. Lett. 95, 070404 (2005).

[7] V. Gurarie and L. Radzihovsky, Ann. Phys. (N.Y.) 322, 2 (2007).

[8] M. Iskin and C. A. R. Sá de Melo, Phys. Rev. Lett. 96, 040402 (2006).

[9] G. E. Volovik, The Universe in a Helium Droplet (Oxford University Press, Oxford, 2003).

[10] J. Zhang, E. G. M. van Kempen, T. Bourdel, L. Khaykovich, J. Cubizolles, F. Chevy, M. Teichmann, L. Tarruell, S. J. J. M. F. Kokkelmans, and C. Salomon, Phys. Rev. A 70, 030702 (2004).

[11] C. Ticknor, C. A. Regal, D. S. Jin, and J. L. Bohn, Phys. Rev. A 69, 042712 (2004).

[12] E. Wille et al., Phys. Rev. Lett. 100, 053201 (2008).

[13] C. Kohstall, M. Zaccanti, M. Jag, A. Trenkwalder, P. Massignan, G. M. Bruun, F. Schreck, and R. Grimm, Nature (London) 485, 615 (2012).

[14] M. Koschorreck, D. Pertot, E. Vogt, B. Fröhlich, M. Feld, and M. Köhl, Nature (London) 485, 619 (2012).

[15] Y. Zhang, W. Ong, I. Arakelyan, and J. E. Thomas, Phys. Rev. Lett. 108, 235302 (2012).

[16] F. Chevy, E. G. M. van Kempen, T. Bourdel, J. Zhang, L. Khaykovich, M. Teichmann, L. Tarruell, S. J. J. M. F. Kokkelmans, and C. Salomon, Phys. Rev. A 71, 062710 (2005).
[17] Otherwise time-reversal symmetry is broken and an additional term appears in the Hamiltonian [7].

[18] In general, $k_{0}$ will also depend on $m_{l}$ through the choice of cut-off function, however, for simplicity we assume $k_{0}$ to be a constant.

[19] J. Levinsen, N. R. Cooper, and V. Gurarie, Phys. Rev. Lett. 99, 210402 (2007).

[20] M. Jona-Lasinio, L. Pricoupenko, and Y. Castin, Phys. Rev. A 77, 043611 (2008).

[21] R. Combescot, A. Recati, C. Lobo, and F. Chevy, Phys. Rev. Lett. 98, 180402 (2007).

[22] P. Massignan and G. M. Bruun, Eur. Phys. J. D 65, 83 (2011).

[23] R. Schmidt and T. Enss, Phys. Rev. A 83, 063620 (2011).

[24] X. Cui and H. Zhai, Phys. Rev. A 81, 041602(R) (2010).

[25] P. Massignan, Europhys. Lett. 98, 10012 (2012).

[26] R. Qi and H. Zhai, Phys. Rev. A 85, 041603(R) (2012).

[27] C. Trefzger and Y. Castin, Phys. Rev. A 85, 053612 (2012).

[28] L.D. Landau and E. M. Lifshitz, Quantum Mechanics (Butterworth-Heinemann, Oxford, UK, 1981).

[29] J. P. Gaebler, J. T. Stewart, J. L. Bohn, and D. S. Jin, Phys. Rev. Lett. 98, 200403 (2007).

[30] J. Fuchs, C. Ticknor, P. Dyke, G. Veeravalli, E. Kuhnle, W. Rowlands, P. Hannaford, and C. J. Vale, Phys. Rev. A 77, 053616 (2008).

[31] Y. Inada, M. Horikoshi, S. Nakajima, M. KuwataGonokami, M. Ueda, and T. Mukaiyama, Phys. Rev. Lett. 101, 100401 (2008).

[32] J. Levinsen, N. R. Cooper, and V. Gurarie, Phys. Rev. A 78, 063616 (2008).

[33] K. Goyal, I. Reichenbach, and I. Deutsch, Phys. Rev. A 82, 062704 (2010).

[34] J. Levinsen, T. Tiecke, J. Walraven, and D. Petrov, Phys. Rev. Lett. 103, 153202 (2009).

[35] Y. Nishida and S. Tan, Phys. Rev. A 82, 062713 (2010). 